\title{
PLASMA BIOMARKERS OF SYSTEMIC REDOX STATUS: A POTENTIAL PREDITOR OF CHEMOTHERAPY. EFFICACY IN WOMEN WITH BREAST CANCER
}

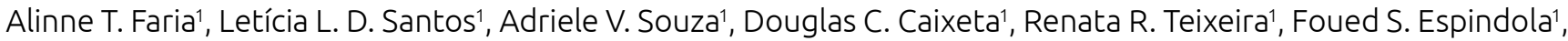
Luiz R. Goulart' ${ }^{1}$ Yara C. P. Maia ${ }^{1}$

${ }^{1}$ Universidade Federal de Uberlândia - Uberlândia (MG), Brazil.

The aim of this study was to verify if the profile of plasma biomarkers of systemic redox status differ between women with breast cancer (BC) submitted or not to chemotherapy (CT), and to associate these results with the CT protocols used in clinical practice. Women with BC were divided in two groups: patients not previously submitted to CT (n=23) and patients previously submitted to $\mathrm{CT}(\mathrm{n}=43)$. Blood samples were collected and plasma was used to perform biochemical analysis to determine the levels of antioxidant enzymes: superoxide dismutase (SOD), catalase (CAT), glutathione reductase (GR), and glutathione peroxidase (GPx), as well as the levels of glutathione tripeptide reduced (GSH). Statistical analyses were carried out using SPSS version 21.0, and Mann Whitney test was performed to verify the differences between groups. Significant differences were found for all activity profiles of the antioxidant enzymes, with a higher enzymatic activity in $\mathrm{BC}$ women who underwent $\mathrm{CT}(\mathrm{p}<0.05)$. This result showing higher production of these enzymes in those women reinforces the adaptive capacities of the organism to CT. Lower levels of GSH were found in CT group ( $\mathrm{p}<0.001)$, especially in those using FAC (5-fluorouracil, adriamycin and cyclophosphamide) protocol. These results may indicate that the regeneration of GSSG to GSH in prooxidative treatment does not remain constant even with the greater production of the GPx and GR enzymes, and that regeneration is subject to interference from other metabolic pathways, such as the pentose phosphate pathway. In addition, the chemotherapeutic protocol (FAC) have been shown to influence on higher oxidative stress. We suggest the investigation of this systemic redox status of women submitted to CT as a relevant conduct to evaluate the efficacy of the chemotherapeutic protocol. 